\title{
Recent advances in the management of venous thromboembolism
}

\author{
Walter Ageno \\ Research Center on Thromboembolic Diseases and Antithrombotic Therapies, Department of Clinical Medicine, University of \\ Insubria, Varese, Italy
}

p-ISSN 1738-7949 / e-ISSN 2092-9129 DOI: $10.5045 / \mathrm{kjh} .2010 .45 .1 .8$ Korean J Hematol 2010;45:8-13.

Received on February 21, 2010 Revised on March 8, 2010 Accepted on March 15, 2010

\begin{abstract}
Venous thromboembolism (VTE) is a spectrum of diseases that includes deep vein thrombosis (DVT) and pulmonary embolism (PE). Anticoagulant treatment is the mainstay of therapy for VTE. Unfractionated heparin (UFH) or low molecular weight heparin (LMWH) followed by vitamin $\mathrm{K}$ antagonists have been the treatment of choice for most patients with VTE, with the aim to prevent thrombus extension or embolization and recurrent VTE. Fondaparinux, a selective, indirect, parenteral factor Xa inhibitor, is now also approved for the initial treatment of VTE and represents an important alternative to UFH or LMWH. Secondary prevention of VTE with vitamin K antagonists is usually prescribed for a minimum of three months, with the duration of treatment based on the presence or absence of major identifiable risk factors for the index event. Patients with permanent risk factors or patients with recurrent DVT or PE require life long secondary prevention. Over the last years, new oral anticoagulant agents have been developed and are now undergoing extensive clinical evaluation in several settings, including the treatment of VTE. New oral anticoagulants include selective, direct thrombin inhibitors, such as dabigatran etexilate, and selective, direct factor Xa inhibitos, such as rivaroxaban, apixaban or edoxaban. All these drugs are admistered at fixed daily doses and do not require laboratory monitoring. The positive results of the first completed clinical trials suggest that a new era in the management of VTE is about to begin.
\end{abstract}

Key Words Deep vein thrombosis, Pulmonary embolism, Anticoagulants, Treatment

\section{STATE OF THE ART IN THE TREATMENT OF VENOUS THROMBOEMBOLISM}

Deep vein thrombosis (DVT) and pulmonary embolism (PE) are important pathologies that affect apparently healthy individuals as well as medical or surgical patients. Therapeutic objectives are essentially the prevention of thrombus extension and embolization, and the prevention of recurrent episodes of venous thromboembolism (VTE) to reduce the risk of fatal pulmonary emboli. Despite the availability of different treatment strategies, the large majority of patients commonly receive a similar therapeutic approach, and the choice of the treatment is eventually influenced by the severity of the presentation of the disease. Anticoagulation is the main therapy for acute VTE and the evidence for the need for anticoagulation in these patients is based on the results of clinical studies performed more than 40 years ago [1]. Patients need to start treatment as soon as the diagnosis is confirmed by objective testing, and because anticoagulant drugs with a rapid onset of action are needed in this phase, three parenteral therapeutic options are currently available for initial treatment: unfractionated heparin (UFH), low-molecular-weight heparin (LMWH), and fondaparinux [2]. Fondaparinux is a synthetic pentasaccharide that inhibits factor Xa indirectly by binding to antithrombin with high affinity and was recommended for the first time in the $8^{\text {th }}$ edition of the American College of Chest Physicians (ACCP) Guidelines on Antithrombotic and Thrombolytic Therapy, which is the most recent and was published in 2008 [2]. This recommendation was based on the results of the MATISSE studies [3, 4]. In the MATISSE DVT study [3], 2205 patients with DVT were treated with a once daily subcutaneous dose of fondaparinux $(7.5 \mathrm{mg}$ for patients weighting 50 to $100 \mathrm{~kg}, 5.0 \mathrm{mg}$ for patients weighting less than $50 \mathrm{~kg}$ and $10.0 \mathrm{mg}$ for patients weighting more than $100 \mathrm{~kg}$ ) or with a twice daily subcutaneous dose of enoxaparin $(1 \mathrm{mg} / \mathrm{kg}$ ) for at least five days. There were no differences in the incidence of recurrent VTE at 3 months $(3.9 \%$ vs $4.1 \%)$, major bleeding while on treatment (1.1\% vs $1.2 \%)$, and mortality at 3 months (3.8\% vs $3.0 \%)$. In the MATISSE $\mathrm{PE}$ study [4], 2213 patients with acute $\mathrm{PE}$ were randomly allocated to treatment with subcutaneous fondaparinux or

This is an Open Access article distributed under the terms of the Creative Commons Attribution Non-Commercial License (http://creativecommons.org/licenses/by-nc/3.0) which permits unrestricted non-commercial use, distribution, and reproduction in any medium, provided the original work is properly cited. 
intravenous UHF. Recurrence of VTE at 3 months $(3.8 \%$ vs $5.0 \%)$ and major bleeding while on treatment $(1.3 \%$ vs $1.1 \%)$ were again similar between the two groups.

In selected cases, more aggressive treatment strategies are required. There is widespread agreement that patients with $\mathrm{PE}$ resulting in cardiogenic shock initially treated with thrombolysis plus anticoagulation have better short- and long-term clinical outcomes than those who receive anticoagulation alone [5]. More recently, some authors have proposed that thrombolysis should be administered to patients with normal blood pressure (and no contraindications) when clinical or echocardiographic evidence of right ventricular dysfunction is present. In the most recent ACCP guidelines [2], the use of thrombolytic therapy, which was previously recommended for hemodynamically unstable patients (massive PE) only, is now also suggested for selected high-risk patients without hemodynamic instability and with a low risk of bleeding, with a grade $2 \mathrm{~B}$ recommendation. However, this remains a controversial issue, and the controversy is likely to remain at least until the results of an ongoing European trial, in which 1,000 PE patients with preserved systolic blood pressure, elevated troponin levels, and right ventricular enlargement on echocardiography are randomised to thrombolytic therapy (tenecteplase plus heparin) versus heparin alone, will become available. Other guidelines, such as those of the European Society of Cardiology, currently do not recommend routine use of thrombolysis in non-high-risk patients [6].

As soon as possible after the diagnosis of VTE, most patients are also started on oral anticoagulant treatment with vitamin $\mathrm{K}$ antagonists for the long-term secondary prevention of the disease. Because of their slow onset of action, and because of their potential to paradoxically increase the prothrombotic state of the patient by also inhibiting endogenous anticoagulants such as protein $\mathrm{C}$, vitamin $\mathrm{K}$ antagonists can not be used as the only treatment strategy during the acute phase of disease and thus require initial association with parenteral anticoagulants for a minimum of 5 days. After this period, oral anticoagulant therapy alone is continued until its benefits (reduction of recurrent VTE) no longer clearly outweigh its risks (increase in bleeding). The risk of recurrence after stopping therapy is largely determined by two factors: whether the acute episode of VTE has been effectively treated; and the patient intrinsic risk of having a new episode of VTE. Therefore, guidelines suggest to treat VTE for at least 3 months if transient risk factors are identified and to consider long-term treatment for patients with unprovoked proximal VTE and no risk factors for bleeding, in whom good quality anticoagulant monitoring is achievable [2]. When the risk to benefit ratio remains uncertain, patient preference to continue or to stop treatment should also be taken into account. VTE is defined unprovoked if cancer or a reversible provoking risk factor is not present. Reversible provoking factors include major risk factors such as surgery, hospitalization, or plaster cast immobilization, if within 1 month; and minor risk factors such as surgery, hospitalization, or plaster cast immobilization, if they have occurred
1 to 3 months before the diagnosis of VTE, and estrogen therapy, pregnancy, or prolonged travel (i.e. more than 8 hours). The greater is the impact of the provoking reversible risk factor (e.g., recent major surgery) on the risk of VTE, the lower is the expected risk of recurrence after stopping anticoagulant therapy. Of interest, in the most recent version of the ACCP guidelines, the presence of thrombophilia is no longer considered for the risk stratification of the patients.

For the secondary prevention of VTE in patients with active cancer, the use of LMWH for the first 3 to 6 months is now preferred over the use of vitamin $\mathrm{K}$ antagonists [2]. This recommendation is based on the results of three studies that selectively enrolled a total of 1,029 patients with VTE in association with active cancer and that found that, compared to oral anticoagulant therapy with vitamin $\mathrm{K}$ antagonists, 3 months or 6 months of therapeutic-dose LMWH was associated with less recurrent VTE in one study and less bleeding in another study (relative risk for the three studies: recurrent VTE, 0.56; 95\% CI, 0.38-0.82; major bleeding, 1.01 ; 95\% CI, 0.62-1.64; mortality, 0.92; 95\% CI, 0.781.10) [7-9]. LMWH is usually administered at full therapeutic dose for the first month and then reduced at approximately $75 \%$ of the initial dose thereafter.

\section{NEW STRAEGIES TO INDIVIDUALIZE THE} DURATION OF SECONDARY PREVENTION

There is a trend toward a more extended duration (potentially life-long) of secondary prevention for a large proportion of patients with a first episode of VTE, namely those with an unprovoked proximal DVT or PE who have a low risk of bleeding and those with a permanent risk factor such as cancer [2]. Indeed, given the high rate of VTE events still defined as unprovoked, which ranges between $26 \%$ and $47 \%$ [10], this recommendation has an enormous potential impact on the long-term management of patients with VTE and on related costs. To overcome this problem, there is an increasing interest in the use of clinical prognostic factors to assist clinicians in individualizing the optimal duration of secondary prevention of unprovoked VTE. These include the measurements of D-dimer and of residual venous obstruction at ultrasound. These strategies, although still not widely accepted, are now supported by the results of randomized clinical trials and of large cohort studies [11-16]. In the PROLONG study [11], patients with unprovoked VTE underwent D-dimer testing 1 month after oral anticoagulant treatment discontinuation. Patients with a normal D-dimer level did not resume anticoagulation, whereas those with an abnormal D-dimer level were randomly assigned either to resume or to discontinue treatment. The D-dimer assay was abnormal in $36.7 \%$ of patients. The rate of recurrences was $15.0 \%$ among the 120 patients who stopped anticoagulation as compared with $2.9 \%$ among the 103 patients who resumed anticoagulation, for an adjusted hazard ratio of 4.26 (95\% confidence interval, 1.23-14.6). VTE recurred in $6.2 \%$ of patients with a normal D-dimer level. Because 
D-dimer levels may increase over time and a single normal D-dimer may be inadequate to predict a low risk of recurrence, the same group carried out a second study, the PROLONG II study, with the aim to assess the time course of $\mathrm{D}$-dimer and its relation with late recurrences in patients with normal D-dimer 1 month after anticoagulation suspension for a first episode of unprovoked VTE [12]. This study showed that when D-dimer becomes abnormal at the third month and remains abnormal afterward, the risk of recurrence is higher than in patients in whom D-dimer remains normal at the third month and afterward (adjusted hazard ratio: 7.9 ; 95\% confidence interval: 2.1-30).

Two randomized controlled studies have evaluated the role of residual vein thrombosis to predict the risk of recurrent VTE $[15,16]$. In the first study, patients with a first episode of DVT were managed according to ultrasound findings after an initial course of oral anticoagulant treatment. Patients with evidence of residual vein thrombosis were randomized to either stop or continue anticoagulants for 9 additional months, whereas patients without residual vein thrombosis treatment was stopped [15]. Residual thrombosis was detected in $69.8 \%$ of patients; recurrent events occurred in $27.2 \%$ of those who discontinued $(15.2 \%$ person-years) and $19.3 \%$ of those who continued oral anticoagulant treatment (10.1\% person-years). The relative adjusted hazard ratio was 1.58 (95\% confidence interval $0.85-2.93)$. Of the $30.2 \%$ patients without residual thrombosis, only $1.3 \%$ ( $0.63 \%$ person-years) had a recurrence. In the second study, 538 patients with a first episode of acute proximal DVT at completion of an uneventful 3-month period of anticoagulation were randomly assigned to fixed-duration anticoagulation (no further anticoagulation for secondary thrombosis and an extra 3 months for unprovoked thrombosis) or flexible-duration, ultrasonography-guided anticoagulation (no further anticoagulation in patients with recanalized veins and continued anticoagulation in all other patients for up to 9 months for secondary DVT and up to 21 months for unprovoked thrombosis) [16]. Overall, 17.2\% of the patients allocated to fixed- duration anticoagulation and $11.9 \%$ of the patients allocated to flexible-duration anticoagulation developed recurrent VTE (adjusted hazard ratio, 0.64; 95\% confidence interval 0.39-0.99). For patients with unprovoked DVT, the adjusted hazard ratio was 0.61 (95\% confidence interval 0.36-1.02) and 0.81 (95\% confidence interval 0.32-2.06) for those with secondary DVT.

\section{NEW ANTICOAGULANTS FOR THE TREATMENT OF VENOUS THROMBOEMBOLISM}

The approach to the development of new anticoagulants as alternatives to heparins and vitamin $\mathrm{K}$ antagonists has been guided by the requirement for convenient administration with predictable pharmacokinetics, pharmacodynamics and a wide therapeutic window that would permit fixed dosing without requiring coagulation monitoring. Research has in particular focussed on targeting thrombin (Factor IIa) and Factor $\mathrm{Xa}$, which are common to both the

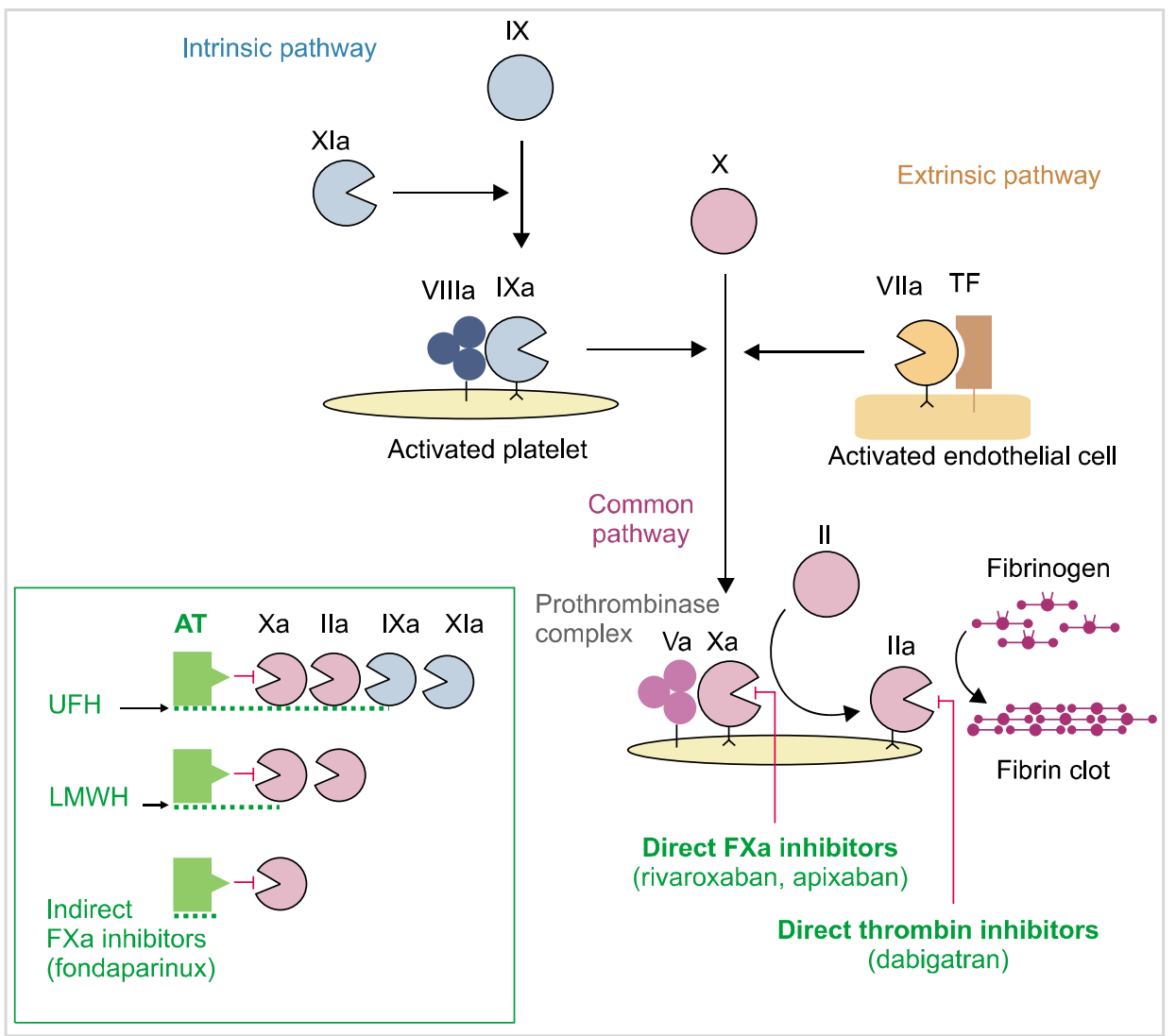

Fig. 1. Mechanism of action of antithrombotic agents in the coagulation pathways. Direct factor Xa (FXa) inhibitors (e.g. Rivaroxaban, Apixaban) are able to inhibit in a selective and reversible manner the active site of both free and prothrombinase-bound FXa. Dabigatran etexilate is an univalent direct thrombin inhibitor that binds exclusively to the active site of thrombin (FIla) to inactivate fibrin-bound thrombin. Binding with antithrombin (AT) respectively, the unfractionated heparin (UFH) inhibits factors Xla, IXa, Xa, and Ila, where as the low-moleculareight heparin (LMWH) inhibits FXa and Flla. Fondaparinux is a synthetic pentasaccharide that inhibits FXa only indirectly by binding to AT with high affinity. 
intrinsic and extrinsic coagulation pathways (Fig. 1). Thrombin inhibitors act to prevent fibrin formation, as well as inhibiting thrombin-mediated activation of Factors V, VIII, $\mathrm{XI}$ and XIII, and platelets. Inhibitors of Factor Xa act at an earlier stage in the cascade, they can inhibit both free and prothrombinase-bound Factor $\mathrm{Xa}$ and are also able to inhibit clot-associated Factor Xa, thus preventing clot-associated Factor Xa from activating prothrombin and thereby contributing to the procoagulant activity of thrombi and therefore to the propagation of the thrombus [17].

\section{Direct thrombin inhibitors}

Dabigatran etexilate is an univalent direct thrombin inhibitor that binds exclusively to the active site of thrombin with the advantage, in comparison with heparins, to inactivate fibrin-bound thrombin. Moreover, dabigatran etexilate is a reversible direct thrombin inhibitor, which dissociates relatively quickly from thrombin, leaving a small amount of free, enzymatically active thrombin available for control of haemostasis. Dabigatran etexilate, is the prodrug of dabigatran, is rapidly absorbed from the gastro-intestinal tract and has a rapid onset of the anticoagulant activity, with plasma levels peak at 2 hours [18]. The half-life ranges between 12 and 17 hours [18]. Dabigatran produces a predictable anticoagulant effect, requires no coagulation monitoring and can be given once daily. It prolongs the activated partial thromboplastin time, but its effect is not dose-linear and it is not suitable for a precise quantification of the anticoagulant effect. At least $80 \%$ of dabigatran is excreted unchanged via the kidneys; therefore, the drug is contraindicated in patients with severe renal failure, with a creatinine clearance less than $30 \mathrm{~mL} / \mathrm{min}$ [18]. Dabigatran etexilate has been already licensed in the European Union and in Canada for the prevention of VTE in patients undergoing hip- and knee-replacement surgery, with a recommended dose of $220 \mathrm{mg}$ once daily for all patients but those with moderate renal insufficiency (creatinine clearence between 30 and $50 \mathrm{~mL} / \mathrm{min}$ ) and the elderly (aged 75 or more), for whom the recommended dose is $150 \mathrm{mg}$ once daily [19]. A dose reduction is also recommended for patients on amiodarone treatment [19].

Dabigatran etexilate is currently undergoing a large phase III program for the evaluation of its efficacy and safety in the acute treatment end in the secondary prevention of VTE. The RE-COVER trial evaluated dabigatran for 6 month treatment of acute symptomatic VTE, while the RE-MEDY and the RE-SONATE trials are recruiting patients who have been successfully treated with standard doses of an approved anticoagulant for three to six months or who have completed 6 to 18 months of treatment with vitamin $\mathrm{K}$ antagonist for confirmed acute symptomatic VTE, respectively. The RECOVER study was published at the end of 2009 [20]. Patients with acute VTE, DVT and/or PE, who were initially treated with parenteral anticoagulants, were randomized to receive dabigatran etexilate, administered at a dose of $150 \mathrm{mg}$ twice daily, or dose adjusted warfarin (international normalized ratio of 2.0 to 3.0 ). The primary outcome of the study was the 6-month incidence of recurrent symptomatic, objectively confirmed VTE and related deaths. Thirty of the 1,274 (2.4\%) dabigatran patients, as compared with 27 of the 1,265 (2.1\%) warfarin patients, had recurrent VTE. The difference in risk was 0.4 percentage points (95\% confidence interval, -0.8 1.5). The hazard ratio with dabigatran was 1.10 (95\% confidence interval, 0.65-1.84). Major bleeding episodes occurred in $20(1.6 \%)$ dabigatran patients and in $24(1.9 \%)$ warfarin patients (hazard ratio with dabigatran, 0.82; $95 \%$ CI, 0.45-1.48), and episodes of any bleeding were observed in 205 (16.1\%) dabigatran patients and in 277 (21.9\%) warfarin patients (hazard ratio with dabigatran, 0.71 ; $95 \%$ confidence interval, 0.59-0.85).

\section{Direct factor Xa inhibitors}

Rivaroxaban is the first of this new class of drugs. It is a potent and selective oral Factor Xa inhibitor with a particular chemical structure in its active-site binding region that plays a role in the oral absorption of the drug, with a relatively high bioavailabity (nearly 80\%) [21]. Plasma levels of the drug peak after 3 to 4 hours, with a mean half-life ranging from 5 to 9 hours in young individuals, and from 11 to 13 hours in the elderly [22]. The main route of excretion is renal, but the drug is also expelled via the faecal/biliar route [23]. Rivaroxaban can be administered at a fixed dose in any patient and does not need laboratory monitoring. Also rivaroxaban has been licensed in the European Union and in Canada for the prevention of VTE in patients undergoing hip- and knee-replacement surgery, with a recommended dose of $10 \mathrm{mg}$ once daily [24].

Two phase II, dose-finding studies compared rivaroxaban administered at total daily doses ranging from $20 \mathrm{mg}$ to $60 \mathrm{mg}$ with standard therapy with LMWH followed by oral vitamin $\mathrm{K}$ antagonists [25, 26]. Based on the positive results of these studies, the following doses were selected for further investigation in the three phase III clinical trials aimed to assess the acute phase and the long term treatment of DVT and PE (the Einstein studies): $15 \mathrm{mg}$ bid for 3 weeks followed by $20 \mathrm{mg}$ qd in the ongoing Einstein DVT and Einstein $\mathrm{PE}$ studies, in which patients with objectively confirmed, symptomatic DVT or PE are randomized to treatment with rivaroxaban alone or with $\mathrm{LMWH}$ and vitamin $\mathrm{K}$ antagonists for a total period of 3 to 12 months, and $20 \mathrm{mg}$ qd in the Einstein Extension study, in which patients who had completed 6 to 12 months of anticoagulant treatment with either vitamin $\mathrm{K}$ antagonists or rivaroxaban (if also enrolled in the acute phase studies) after an acute episode of VTE were randomized to rivaroxaban or placebo for additional 6 to 12 months. The Einstein Extension study is already completed, and the results have been presented at the American Society of Hematology meeting in December 2009 [27]. In this randomised, double blind, placebo-controlled study, the primary efficacy outcome was the recurrence of symptomatic VTE and the principal safety outcome was the occurrence of major bleeding. During treatment, symptomatic recurrent VTE events occurred in $7.1 \%$ patients treated with placebo and in $1.3 \%$ patients treated with rivaroxaban (hazard ratio, 
0.18; 95\% confindence interval, 0.09-0.39). After stopping the study medication, $1.0 \%$ symptomatic recurrent VTE events occurred in both groups during the one month observational period of follow up. No major bleeding events were documented in the group of patients treated with placebo, $4(0.7 \%)$ major bleeding events occurred in the rivaroxaban group $(P=0.106)$. None of these bleeding events were fatal or occurred in a critical site. Clinically relevant non-major bleeding occurred in $1.2 \%$ and in $5.4 \%$ patients randomized to placebo and rivaroxaban, respectively. Two (0.3\%) patients in the placebo group and $1(0.2 \%)$ patient in the rivaroxaban group died.

Apixaban is an oral active Factor Xa inhibitor derived from razaxaban (an aminobenzisoxazole that binds to the active site of Factor Xa with high affinity), with superior pharmacological proprieties [17]. It is a small molecule able to inhibit in a selective and reversible manner the active site of both free and prothrombinase-bound Factor Xa. Preclinical studies demonstrate that apixaban has an oral bioavailability of more than $50 \%$ : its plasma peak is achieved in about $3 \mathrm{~h}$ and its half-life is about $12 \mathrm{~h}$ [28]. The drug is absorbed in the gastrointestinal tract, is metabolised in the liver by cythocrome-dependent and -independent mechanisms and it is eliminated through both the renal and the faecal routes [29].

Apixaban has been assessed for the treatment of DVT in a dose finding study (Botticelli DVT study) [30]. Patients were randomised to receive apixaban $5 \mathrm{mg}$ bid, $10 \mathrm{mg}$ bid, $20 \mathrm{mg}$ od or LMWH vitamin $\mathrm{K}$ antagonists. The primary efficacy outcome, defined as the composite of symptomatic recurrent VTE and asymptomatic deterioration in the thrombotic burden as assessed by repeat bilateral compression ultrasonography and perfusion lung scan, occurred in $4.7 \%$ of patients treated with apixaban and in $4.2 \%$ of LMWH/vitamin $\mathrm{K}$ antagonists treated patients. No dose effect was observed across apixaban doses. The principal safety outcome, defined as the composite of major and clinically relevant non-major bleeding, occurred in $7.3 \%$ of the apixaban treated patients and in $7.9 \%$ of $\mathrm{LMWH} /$ vitamin $\mathrm{K}$ antagonists treated patients. On the basis of this study, phase III studies (AMPLIFY and AMPLIFY extension), testing apixaban at the doses of $10 \mathrm{mg}$ and $5 \mathrm{mg}$ twice daily, are now undergoing. Studies assessing the efficacy and safety of other factor $\mathrm{Xa}$ inhibitors, such as edoxaban, are also underway.

\section{CONCLUSIONS}

The current management of VTE is largely based on the use of anticoagulant drugs, both parenteral drugs such as UFH, LMWH or fondaparinux for the treatment of the acute phase and oral drugs such as the vitamin $\mathrm{K}$ antagonists for the long term secondary prevention. All these drugs have been proven to be highly effective in preventing thrombus propagation, embolization, and recurrence. For the management of the acute phase of the disease, LMWH has largely replaced UFH thus contributing to simplify the management of VTE, and now a large proportion of patients with DVT do not need to be hospitalized and can be entirely treated as outpatients. For the long term secondary prevention, vitamin $\mathrm{K}$ antagonists remain the only choice for clinicians, and their clear benefits in terms of efficacy need to be periodically balanced in each patient against their risks in terms of safety and their inconvenient management. In a very near future, the armamentarium of clinicians involved in the prevention and treatment of thromboembolic disorders could become much larger. After the positive results of the first clinical trials, new direct thrombin inhibitors and direct Factor Xa inhibitors that are administered orally are closely approaching the market. With predictable anticoagulant responses and low potential for food-drug and drug-drug interactions, these new agents can be given in fixed doses without coagulation monitoring. These properties and the oral administration render these compounds more convenient than both vitamin $\mathrm{K}$ antagonists and LMWH. Based on design of the phase III clinical trials, we can speculate that some of these compounds will challenge the vitamin $\mathrm{K}$ antagonists for the long term secondary prevention of VTE, and that other will also challenge the parenteral drugs for the acute phase management, as they are tested as a stand-alone treatment for both DVT and PE. Thus, patients with VTE could be treated with a single oral agent right after the objective diagnosis of the disease. Specific areas of particular interest for these new agents include the treatment of patients with cancer and VTE, for whom long term treatment with LMWH is currently recommended and for whom an oral agent with a low propensity for drug-drug interactions could represent the ideal therapy, and of course the long term treatment of patients with unprovoked VTE, where the complex balance between benefits and risks of the currently available drugs could be simplified with the use of more practical agents.

\section{REFERENCES}

1. Barritt DW, Jordan SC. Anticoagulant drugs in the treatment of pulmonary embolism: a controlled trial. Lancet 1960;1:1309-12.

2. Kearon C, Kahn SR, Agnelli G, et al. Antithrombotic therapy for venous thromboembolic disease: american college of chest physicians evidence-based clinical practice guidelines (8th Edition). Chest 2008;133(6 Suppl):454S-545S.

3. Büller HR, Davidson BL, Decousus H, et al. Fondaparinux or enoxaparin for the initial treatment of symptomatic deep venous thrombosis: a randomized trial. Ann Intern Med 2004;140: 867-73.

4. Büller HR, Davidson BL, Decousus H, et al. Subcutaneous fondaparinux versus intravenous unfractionated heparin in the initial treatment of pulmonary embolism. N Engl J Med 2003;349:1695702.

5. Konstantinides S, Geibel A, Heusel G, et al. Heparin plus alteplase compared with heparin alone in patients with submassive pulmonary embolism. N Engl J Med 2002;347:1143-50.

6. Torbicki A, Perrier A, Konstantinides S, et al. Guidelines on the 
diagnosis and management of acute pulmonary embolism: the task force for the diagnosis and management of acute pulmonary embolism of the european society of cardiology (ESC). Eur Heart J 2008;29:2276-315.

7. Lee AY, Levine MN, Baker RI, et al. Low-molecular-weight heparin versus a coumarin for the prevention of recurrent venous thromboembolism in patients with cancer. N Engl J Med 2003; 349:146-53.

8. Meyer G, Marjanovic Z, Valcke J, et al. Comparison of low-molecular-weight heparin and warfarin for the secondary prevention of venous thromboembolism in patients with cancer: a randomized controlled study. Arch Intern Med 2002; 162:1729-35.

9. Hull RD, Pineo GF, Brant RF, et al. Long-term low-molecular-weight heparin versus usual care in proximal-vein thrombosis patients with cancer. Am J Med 2006;119:1062-72.

10. White RH. The epidemiology of venous thromboembolism. Circulation 2003;107:I4-8.

11. Palareti G, Cosmi B, Legnani C, et al. D-dimer testing to determine the duration of anticoagulation therapy. N Engl J Med 2006;355: 1780-9.

12. Cosmi B, Legnani C, Tosetto A, et al. Usefulness of repeated D-dimer testing after stopping anticoagulation for a first episode of unprovoked venous thromboembolism: the PROLONG II prospective study. Blood 2010;115:481-8.

13. Eichinger S, Minar E, Bialonczyk C, et al. D-dimer levels and risk of recurrent venous thromboembolism. JAMA 2003;290:1071-4.

14. Prandoni P, Lensing AW, Prins MH, et al. Residual venous thrombosis as a predictive factor of recurrent venous thromboembolism. Ann Intern Med 2002;137:955-60.

15. Siragusa S, Malato A, Anastasio R, et al. Residual vein thrombosis to establish duration of anticoagulation after a first episode of deep vein thrombosis: the Duration of Anticoagulation based on Compression UltraSonography (DACUS) study. Blood 2008;112: 511-5.

16. Prandoni P, Prins MH, Lensing AW, et al. Residual thrombosis on ultrasonography to guide the duration of anticoagulation in patients with deep venous thrombosis: a randomized trial. Ann Intern Med 2009;150:577-85.

17. Turpie AGG. Oral, direct Factor Xa inhibitors in development for the prevention and treatment of thromboembolic diseases. Arterioscler Thromb Vasc Biol 2007;27:1238-47.

18. Weitz JI, Hirsh J, Samama MM. New antithrombotic drugs: American college of chest physicians evidence-based clinical practice guidelines (8th Edition). Chest 2008;133:234S-56S.

19. European public assessment report: Pradaxa. London: European
Medicines Agency (EMEA), 2008. (Accessed January 10, 2010, at http://www.emea.europa.eu)

20. Schulman S, Kearon C, Kakkar AK, et al. Dabigatran versus warfarin in the treatment of acute venous thromboembolism. $\mathrm{N} \mathrm{Engl} \mathrm{J}$ Med 2009;361:2342-52.

21. Kubitza D, Becka M, Voith B, Zuehlsdorf M, Wensing G. Safety pharmacodynamics and pharmacokinetics of single doses of BAY 59-7939, an oral, direct factor Xa inhibitor. Clin Pharmacol Ther 2005;78:412-21.

22. Eriksson BI, Quinlan DJ, Weitz JI. Comparative pharmacodynamics and pharmacokinetics of oral direct thrombin and factor Xa inhibitors in development. Clin Pharmacokinet 2009;48:1-22.

23. Kubitza D, Becka M, Wensing G, Voith B, Zuehlsdorf M. Safety pharmacodynamics and pharmacokinetics of BAY59-7939-an oral, direct Factor Xa inhibitor-after multiple dosing in healthy male subjects. Eur J Clin Pharmacol 2005;61:873-80.

24. European public assessment report: Xarelto. London: European Medicines Agency (EMEA), 2008. (Accessed January 10, 2010, at http://www.emea.europa.eu)

25. Agnelli G, Gallus A, Goldhaber SZ, et al. Treatment of proximal deep-vein thrombosis with the oral direct factor Xa inhibitor rivaroxaban (BAY59-7939): the ODIXa DVT (oral direct factor Xa inhibitor BAY59-7939 in patients with acute symptomatic deep-vein thrombosis) study. Circulation 2007;116:180-7.

26. Buller HR, Lensing AW, Prins MH, et al. A dose-ranging study evaluating once-daily oral administration of the factor Xa inhibitor rivaroxaban in the treatment of patients with acute symptomatic deep vein thrombosis: the Einstein-DVT Dose-Ranging Study. Blood 2008;112:2242-7.

27. Buller HR. Once daily oral rivaroxaban versus placebo in the long term prevention of recurrent symptomatic venous thromboembolism. The Einstein-Extension study. Blood 2009;114:Abstract 2.[U01]

28. Wong PC, Crain EJ, Xin B, et al. Apixaban, an oral, direct and highly selective factor Xa inhibitor: in vitro, antithrombotic and antihemostatic studies. J Thromb Haemost 2008;6:820-9.

29. Wang L, Zhang D, Raghavan N, et al. In vitro assessment of metabolic drug-drug interaction potential of apixaban through cytochrome P450 phenotyping, inhibition, and induction studies. Drug Metab Dispos 2010;38:448-58.

30. Buller H, Deitchman D, Prins M, et al. Efficacy and safety of the oral direct facto Xa inhibitor apixaban for symptomatic deep vein thrombosis. The Botticelli DVT dose-ranging study. J Thromb Haemost 2008;6:1313-8. 